Pacific Journal of Mathematics

UNITARY BORDISM OF CLASSIFYING SPACES OF 


\section{UNITARY BORDISM OF CLASSIFYING SPACES OF QUATERNION GROUPS}

\section{Abdeslam Mesnaoui}

Let $\Gamma_{k}$ be the generalized quaternion group of order $2^{k}$. In this article we determine a set of generators for the $U_{*}(p t)$-module $\widetilde{U}_{*}\left(B \Gamma_{k}\right)$ and give all linear relations between them. Moreover their orders are calculated.

0. Introduction. In this article we first study the case $\Gamma_{k}=\Gamma$ the quaternion group of order 8 . We recall that

$\Gamma=\{ \pm 1, \pm i, \pm j, \pm k\}, \quad i^{2}=j^{2}=k^{2}=-1, i j=k, j k=i, k i=i j$.

$\Gamma$ acts on $S^{4 n-3}$ by using $(n+1) \eta$ where $\eta$ denotes the following unitary irreducible representation of $\Gamma: i \rightarrow\left(\begin{array}{cc}i & 0 \\ 0 & -i\end{array}\right), j \rightarrow\left(\begin{array}{cc}0 & -1 \\ 1 & 0\end{array}\right)$ and we get the element $w_{4 n+3}=\left[S^{4 n+3} / \Gamma, q\right] \in \widetilde{U}_{4 n+3}(B \Gamma), q$ being the natural embedding: $S^{4 n+3} / \Gamma \subset B \Gamma$. In [6] we have defined three elements of $\widetilde{U}^{2}(B \Gamma)$ denoted by $A, B, C$ as Euler classes for $M U$ of irreducible representations of $\Gamma$ of dimension 1 over $\mathbb{C}$. Let $u_{4 n+1} \in \widetilde{U}_{4 n+1}(B \Gamma)$, $v_{4 n+1} \in \widetilde{U}_{4 n+1}(B \Gamma)$ be respectively $A \cap w_{4 n+3}$ and $B \cap w_{4 n+3}$. Our first result is:

TheOREM 2.2. The set $\left\{u_{4 n+1}, v_{4 n+1}, w_{4 n+3}\right\}_{n \geq 0}$ is a system of generators for the $U_{*}(p t)$-module $\widetilde{U}_{*}(B \Gamma)$.

Their orders are given by:

THEOREM 2.6. We have: ord $w_{4 n+3}=2^{2 n+3}$.

THEOREM 2.8. We have: ord $u_{4 n+1}=\operatorname{ord} v_{4 n+1}=2^{n+1}$.

Now let $\Omega_{*}$ be $U^{*}(p t)[[Z]]$ graded by taking $\operatorname{dim} Z=4$. If $P(Z)=\sum_{i \geq r} \alpha_{i} Z^{i} \in \Omega_{n}$ and $\alpha_{r} \neq 0$ then we denote $\nu(P)=4 r$. Let $W, V_{1}, V_{2}$ be the submodules of $\widetilde{U}_{*}(B \Gamma)$ generated respectively by $\left\{w_{4 n+3}\right\}_{n \geq 0},\left\{u_{4 n+1}\right\}_{n \geq 0},\left\{v_{4 n+1}\right\}_{n \geq 0}$. The following result gives the $U_{*}(p t)$-module structure of $\widetilde{U}_{*}(B \Gamma)$ and uses the elements $T(Z) \in \Omega_{4}$, $J(Z) \in \Omega_{0}$ as defined in [6], Section II. 
THEOREM 2.4. (a) $\widetilde{U}_{*}(B \Gamma)=W \oplus V_{1} \oplus V_{2}$.

(b) In $\widetilde{U}_{2 p+1}(B \Gamma)$ we have $0=a_{0} w_{3}+a_{1} w_{7}+\cdots+a_{n} w_{4 n+3}=b_{0} u_{1}+$ $\cdots+b_{m} u_{4 m+1}$ iff there are homogeneous polynomials $M(Z), M_{2}(Z)$ and homogeneous formal power series $N(Z), N_{1}(Z)$ of $\Omega_{*}$ satisfying. $b_{m} Z+b_{m-1} Z^{2}+\cdots+b_{0} Z^{m+1}=M(Z)(2+J(Z))+N(Z), a_{n} Z+$ $a_{n-1} Z^{2}+\cdots+a_{0} Z^{n+1}=M_{1}(Z) T(Z)+N_{1}(Z), \nu(N)>4(n+1)$, $\nu\left(N_{1}\right)>4(n+1)$. Moreover $b_{0} u_{1}+\cdots+b_{m} u_{4 m+1}=0$ iff $b_{0} v_{1}+\cdots+$ $b_{m} v_{4 m+1}=0$.

In Section III we consider $\widetilde{U}_{*}\left(B \Gamma_{k}\right), k \geq 4$. The generalized quaternion group $\Gamma_{k}$ is generated by $u, v$ with $u^{t}=v^{2}, t=2^{k-2}, u v u=v$. $\Gamma_{k}$ acts on $S^{4 n+3}$ by means of the irreducible unitary representation $\eta_{1}$ of $\Gamma_{k}$ :

$$
u \rightarrow\left(\begin{array}{cc}
\omega & 0 \\
0 & \omega^{-1}
\end{array}\right), \quad v \rightarrow\left(\begin{array}{cc}
0 & -1 \\
1 & 0
\end{array}\right),
$$

$\omega$ being a primitive $2^{k-1}$ th root of unity. We get:

$$
w_{4 n+3}^{\prime}=\left[S^{4 n+3} / \Gamma_{k}, q^{\prime}\right] \in \widetilde{U}_{4 n+3}\left(B \Gamma_{k}\right), \quad q^{\prime}: S^{4 n+3} / \Gamma_{k} \subset B \Gamma_{k} .
$$

Now we use the elements $B_{k}^{\prime}=B_{k}+G_{k}\left(D_{k}\right) \in \widetilde{U}^{2}\left(B \Gamma_{k}\right), C_{k}^{\prime}=C_{k}+$ $G_{k}\left(D_{k}\right) \in \widetilde{U}^{2}(B \Gamma)$ (see [6], Theorem 3.14) to define $u_{4 n+1}^{\prime}=B_{k}^{\prime} \cap$ $w_{4 n+3}^{\prime} \in \widetilde{U}_{4 n+1}\left(B \Gamma_{k}\right), v_{4 n+1}^{\prime}=C_{k}^{\prime} \cap w_{4 n+3}^{\prime} \in \widetilde{U}_{4 n+1}\left(B \Gamma_{k}\right)$. Then we have Theorems 3.1, 3.2 identical respectively to the above Theorems $2.2,2.4$ where $w_{4 n+3}, u_{4 n+1}, v_{4 n+1}$ are replaced by $w_{4 n+3}^{\prime}, u_{4 n+1}^{\prime}, v_{4 n+1}^{\prime}$. However:

THEOREM 3.4. We have: ord $w_{4 n+3}^{\prime}=2^{2 n+k}, n \geq 0$.

THEOREM 3.5. We have: ord $u_{4 n+1}^{\prime}=\operatorname{ord} v_{4 n+1}^{\prime}=2^{n+1}, n \geq 0$, which are therefore independent of $k$.

The layout is as follows:

I Preliminaries and notations.

II Calculations in $\widetilde{U}_{*}(B \Gamma)$ : generators, orders and relations.

III $\widetilde{U}_{*}\left(B \Gamma_{k}\right), k \geq 4$ : generators, orders and relations.

We assume that the reader is acquainted with the notations and results of [6].

I. Preliminaries and notations. The notation $U_{*}$-AHSS will be used for the Atiyah-Hirzebruch spectral sequence corresponding to the homology theory determined by $M U ; \mu$ and $\mu^{\prime}$ denote the edge homomorphisms $U^{*}(X) \rightarrow H^{*}(X)$ and $U_{*}(X) \rightarrow H_{*}(X)$ obtained from the 
$U_{*}$-AHSS for a CW complex $X$. We have the following well-known result:

THEOREM 1.1. Suppose $X$ a $C W$-complex such that:

(a) The $U_{*}$-AHSS for $X$ collapses.

(b) For each $n \geq 0$ there is a system $\left(a_{i n}\right)$ generating the group $H_{n}(X)$.

Then for each $n \geq 0$ there is a system $\left(A_{i n}\right)$ such that:

(a) $A_{\text {in }} \in U_{n}(X), \mu^{\prime}\left(A_{\text {in }}\right)=a_{\text {in }}$ for every $(i, n)$.

(b) The system $\left(A_{\text {in }}\right)$ generates $U_{*}(X)$ as a $U_{*}(p t)$-module.

Moreover, (b) is valid for every system $\left(A_{i n}\right)$ such that $\mu^{\prime}\left(A_{i n}\right)=a_{i n}$.

Consider the map of ring spectra $f: M U \rightarrow H$ (see [1]); by naturality of spectral sequences it follows that if $X$ is a CW-complex then $f^{\#}(X)=\mu$ and $f_{\#}(X)=\mu^{\prime}$ where $f^{\#}(X): U^{*}(X) \rightarrow H^{*}(X)$, $f_{\#}(X): U_{*}(X) \rightarrow H_{*}(X)$ denote the maps induced by $f$.

Proposition 1.2. If $X$ is a $C W$-complex then the following diagram commutes:

$$
\begin{array}{ccc}
U^{m}(X) \otimes U_{n}(X) \stackrel{\cap}{\longrightarrow} U_{n-m}(X) \\
\mu \otimes \mu^{\prime} \downarrow & \downarrow^{\prime} \\
H^{m}(X) \otimes H_{n}(X) \stackrel{\cap}{\longrightarrow} H_{n-m}(X) \text { commutes. }
\end{array}
$$

Proof. Take $E=M U$. The cap product is the composite:

$\widetilde{E}_{m}\left(X^{+}\right) \otimes \widetilde{E}_{n}\left(X^{+}\right) \stackrel{1 \otimes \Delta_{*}}{\longrightarrow} \widetilde{E}^{m}\left(X^{+}\right) \otimes \widetilde{E}_{n}\left(X^{+} \wedge X^{+}\right) \stackrel{\backslash}{\longrightarrow} \widetilde{E}_{n-m}\left(X^{+}\right)$, $\backslash$ being the slant product and $\Delta(X)=[x, x]$. Since $\Delta_{*}$ commutes with $f_{\#}(-)$ we have to prove that the diagram:

$$
\begin{array}{cccc}
\widetilde{E}^{m}\left(X^{+}\right) \otimes \widetilde{E}_{n}\left(X^{+} \wedge X^{+}\right) & \perp & \widetilde{E}_{n-m}\left(X^{+}\right) \\
& \downarrow f^{*}(-) \otimes f_{*}(-) & & \downarrow f_{*}(-) \\
\widetilde{H}^{m}\left(X^{+}\right) \otimes \widetilde{H}_{n}\left(X^{+} \wedge X^{+}\right) & \perp & \widetilde{H}_{n-m}\left(X^{+}\right) \text {commutes. }
\end{array}
$$

More generally the diagram

$$
\begin{aligned}
& \widetilde{E}^{m}(Y) \otimes \widetilde{E}_{n}(Y \wedge Z) \stackrel{\backslash}{\longrightarrow} \widetilde{E}_{n-m}(Z) \\
& \downarrow f^{*}(-) \otimes f_{*}(-) \quad \downarrow f_{*}(-) \\
& \widetilde{H}^{m}(Y) \otimes \widetilde{H}_{n}(Y \wedge Z) \stackrel{\backslash}{\longrightarrow} \widetilde{H}_{n-m}(Z) \text { commutes if } Y, Z
\end{aligned}
$$


are pointed $\mathrm{CW}$-complexes: indeed let $x$ and $y$ be any elements of $\widetilde{E}^{m}(Y)$ and $\widetilde{E}_{n}(Y \wedge Z)$ respectively represented by $g: Y \rightarrow \sum^{m} E$, $h: S^{n} \rightarrow E \wedge Y \wedge Z$. Then $f^{\#}(-)(x)$ is represented by the composite

$$
g_{1}: Y \stackrel{g}{\longrightarrow} \sum^{m} E \stackrel{\sum^{m} f}{\longrightarrow} \sum^{m} H \text { and } f_{\#}(-)(y)
$$

by the composite:

$$
h_{1}: S^{n} \stackrel{h}{\longrightarrow} E \wedge Y \wedge Z \stackrel{f \wedge 1 \wedge 1}{\longrightarrow} H \wedge Y \wedge Z .
$$

If we denote by $T$ the transposition and $k, k^{\prime}$ the ring-spectra products then the diagram pictured on the next page commutes. Since the top line represents $x \backslash y$ and the bottom line

$$
f^{\neq}(-)(x) \backslash f_{\neq}(-)(y)
$$

we have $f_{\neq}(-)(x \backslash y)=f^{\neq}(-)(x) \backslash f_{\neq}(-)(y)$.

Let $X$ be any $\mathrm{CW}$-complex and $\xi$ a complex vector bundle of $\mathbb{C}$ dimension $n$ over $X$. If $h$ denotes a map: $X \rightarrow B U(n)$ classifying $\xi$ and $M(\xi)$ the Thom space of $\xi$, then $M(h): M(\xi) \rightarrow M U(n)$ determines an element $t_{0}(\xi) \in U^{2 n}(M(\xi))$ which is a particular Thom class for $\xi$ called the canonical Thom class for $\xi$. Moreover if $j: X \rightarrow M(\xi)$ is the zero section we have $j^{*}\left(t_{0}(\xi)\right)=c f_{n}(\xi)$, the highest ConnerFloyd characteristic class of $\xi ; j^{*}\left(t_{0}(\xi)\right)$ is also called the Euler class $e(\xi)$ of $\xi$.

Fundamental classes for a $U$-manifold $M^{n}$ for $E=M U$ or $H$ may be obtained in the following manner: $M^{n}$ can be embedded in $S^{n+2 k}$ for some large $k$ and the normal bundle $\tau$ can be given a $U(k)$ structure; let $N$ be a tubular neighbourhood of $M^{n}$, which we identify with the total space of the normal disk bundle $D(\tau)$; we have the map $\pi: S^{n+2 k} \rightarrow M(\tau)$ defined as follows: if $x \in N$ then $\pi(x)$ is the image of $x$ by the projection $D(\tau) \rightarrow M(\tau)$ and if $x \in S^{n+2 k}-\stackrel{\circ}{N}$, then $\pi(x)=*$ the base point of $M(\tau)$; let $t$ be a Thom class of $\xi$ for $E$; we have the Thom-isomorphism $\phi_{t}: E_{2 k+r}(M(\tau)) \rightarrow E_{r}\left(M^{n}\right)$ such that $\phi_{t}(x)=p_{*}(t \cap x), p$ being the projection $D(\tau) \rightarrow M^{n}$; let $u: S^{0} \rightarrow E$ be the unit of $E$; the map $u$ is a map of spectra and is therefore a collection of maps $u_{m}: S^{m} \rightarrow E_{m}$ satisfying well-known axioms; then by [8], page 333, if [ $\left.u_{n+2 k}\right]$ is the element of $\widetilde{E}_{n+2 k}\left(S^{n+2 k}\right)$ corresponding to $u_{n+2 k}$, then the element $c(M)=\phi_{t}\left(\pi_{*}\left(\left[u_{n+2 k}\right]\right)\right) \in E_{n}\left(M^{n}\right)$ is a fundamental class for $M^{n}$. Evidently the same method produces fundamental classes for the homology theory defined by the spectrum $H$. 


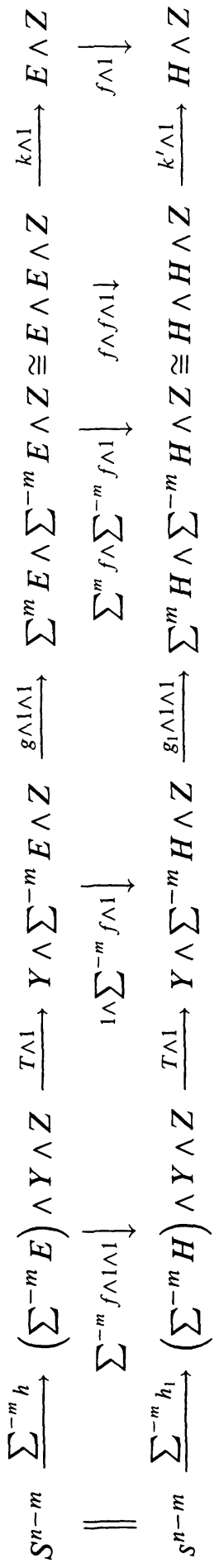


From [8], page 335, §14-45, we have:

Proposition 1.3. If $M^{n}$ is a closed $U$-manifold then $\left[M^{n}, 1\right] \in$ $U_{n}\left(M^{n}\right)=E_{n}\left(M^{n}\right)$ is a fundamental class for $M^{n}$ deduced from the canonical Thom class $t_{0}(\tau), \tau$ being the normal bundle of an embedding $M^{n} \subset S^{n+2 k}, k$ large.

Proposition 1.4. Let $M^{n}$ be a closed $U$-manifold; then

$$
f_{\#}(-)\left(\left[M^{n}, 1\right]\right) \in H_{n}\left(M^{n}\right)
$$

is a fundamental class for $M^{n}$.

Proof. From 1.3 we have

$$
\left[M^{n}, 1\right]=\phi_{t_{0}}\left(\pi_{*}\left[u_{n+2 k}\right]\right)=c(M)
$$

then

$$
\begin{aligned}
f_{\#}(-)(c(M)) & =f_{\#}(-)\left[\phi_{t_{0}}\left(\pi_{*}\left(\left[u_{n+2 k}\right]\right)\right)\right]=f_{\#}(-)\left[p_{*}\left(t_{0} \cap \pi_{*}\left(\left[u_{n+2 k}\right]\right)\right)\right] \\
& =p_{*}\left[f_{\#}(-)\left(t_{0} \cap \pi_{*}\left(\left[u_{n+2 k}\right]\right)\right)\right] \\
& =p_{*}\left[f^{\#}(-)\left(t_{0}\right) \cap f_{\#}(-)\left(\pi_{*}\left(\left[u_{n+2 k}\right]\right)\right)\right] \\
& =p_{*}\left[f^{\#}(-)\left(t_{0}\right) \cap \pi_{*}\left(f(-)\left(\left[u_{n+2 k}\right]\right)\right)\right] .
\end{aligned}
$$

Since $f$ is a map of spectra the unit of $H$ is the composite $v: S^{0} \stackrel{u}{\rightarrow}$ $M U \stackrel{f}{\rightarrow} H$ and hence $f_{\#}(-)\left(\left[u_{n+2 k}\right]\right)=\left[v_{n+2 k}\right]$. Now $f^{\#}(-)\left(t_{0}\right)$ is a Thom class $t_{1}$ for $H$ and therefore

$$
\begin{aligned}
f_{\#}(-)(c(M)) & =p_{*}\left[t_{1} \cap \pi_{*}\left(\left[v_{n+2 k}\right]\right)\right] \\
& =\phi_{t_{1}}\left(\pi_{*}\left(\left[v_{n+2 k}\right]\right)\right)=c_{1}\left(M^{n}\right) \in H_{n}\left(M^{n}\right)
\end{aligned}
$$

is a fundamental class for $M^{n}$.

The notation $c\left(M^{n}\right)$ will be for the fundamental class $\left[M^{n}, 1\right] \in$ $U_{n}\left(M^{n}\right)$ and $c_{1}\left(M^{n}\right) \in H_{n}\left(M^{n}\right)$ will be the fundamental class $\mu^{\prime}\left(c\left(M^{n}\right)\right)$.

If $\mathrm{PD}$ or $\mathrm{PD}_{1}$ denotes the Poincare duality then we have:

Proposition 1.5. The following diagram commutes

$$
\begin{array}{ccc}
U^{m}\left(M^{n}\right) & \stackrel{\mathrm{PD}}{\longrightarrow} & U_{n-m}\left(M^{n}\right) \\
\downarrow^{\mu} & & \downarrow \mu^{\prime} \\
H^{m}\left(M^{n}\right) & \stackrel{\mathrm{PD}_{1}}{\longrightarrow} & H_{n-m}\left(M^{n}\right)
\end{array}
$$


Proof. We have

$$
\begin{aligned}
\mu^{\prime}(\mathrm{PD}(x)) & =\mu^{\prime}\left(x \cap c\left(M^{n}\right)\right)=\mu(x) \cap \mu^{\prime}\left(c\left(M^{n}\right)\right) \\
& =\mu(x) \cap c_{1}\left(M^{n}\right)=(\mathrm{PD})_{1}(\mu(x))
\end{aligned}
$$

by 1.2 .

Let $N^{m}$ be a closed $U$-submanifold of a closed $U$-manifold $M^{n}$, and $i$ the inclusion $N^{m} \subset M^{n}$; then the normal bundle $\tau$ of $N^{m}$ in $M^{n}$ is a complex-vector-bundle if $(n-m)$ is even and we have:

Proposition 1.6. If $(n-m)$ is even then $(\mathrm{PD})^{-1}\left(\left[N^{m}, i\right]\right)$ is represented by:

$$
M^{n} \rightarrow M^{n} /\left(M^{n}-\stackrel{\circ}{N}\right) \simeq D(\tau) / S(\tau)=M(\tau) \stackrel{M(h)}{\longrightarrow} M U\left(\frac{1}{2}(n-m)\right),
$$

where $h$ is a map classifying $\tau$ and $N$ a tubular neighborhood of $N^{m}$ homeomorphic to $D(\tau)$ (see [3], [7]).

The generalized quaternion group $\Gamma_{k}, k \geq 4$, is generated by $u, v$ subject to the relations $u^{t}=v^{2}, t=2^{k-2}, u v u=v$. Consider the irreducible unitary representation $\eta_{1}$ of $\Gamma_{k}: u \rightarrow\left(\begin{array}{cc}\omega & 0 \\ 0 & \omega^{-1}\end{array}\right), v \rightarrow\left(\begin{array}{cc}0 & -1 \\ 1 & 0\end{array}\right)$, $\omega$ being a primitive $2^{k-1}$ th-root of unity. The group $\Gamma_{k}$ acts on $S^{4 n+3}$ by means of $(n+1) \eta_{1}$ as a group of $U$-diffeomorphisms and we get a canonical $U$-structure on $S^{4 n+3} / \Gamma_{k}$ and a natural injection $S^{4 n+3} / \Gamma_{k} \subset$ $B \Gamma_{k}=\bigcup_{n \geq 0} S^{4 n+3} / \Gamma_{k}$ (see [3], [10], page 508).

Let $\alpha$ be the complex vector bundle: $S^{4 n+3} \times_{\Gamma_{k}} \mathbb{C}^{2} \rightarrow S^{4 n+3} / \Gamma_{k}$ where $\Gamma_{k}$ acts on $S^{4 n+3}$ and $\mathbb{C}^{2}$ respectively by means of $(n+1) \eta_{1}$ and $\eta_{1}$ : if $a \in \Gamma_{k}$ and $(x, v) \in S^{4 n+3} \times \mathbb{C}^{2}$ we have $a(s, w)=(a s, a v)=\left(s a^{-1}, a v\right)$ and $S^{4 n+3} \times \Gamma_{k} \mathbb{C}^{2}=\left(S^{4 n+3} \times C^{2}\right) / \Gamma_{k}$. Then by a result of R. H. Szczarba ([9]) we have $T\left(S^{4 n+3} / \Gamma_{k}\right)+1=(n+1) \alpha$ where $T\left(S^{n+3} / \Gamma_{k}\right)$ denotes the tangent bundle of $S^{4 n=3} / \Gamma_{k}$. As an easy consequence we have:

Proposition 1.7. If $i$ denotes the embedding $S^{4 n+3} / \Gamma_{k} \subset S^{4 n+7} / \Gamma_{k}$ such that

$$
i\left(\left[z_{1}, z_{2}, \ldots, z_{2 n+2}\right]\right)=\left[z_{1}, z_{2}, \ldots, z_{2 n+2}, 0,0\right]
$$

then the normal bundle of $S^{4 n+3} / \Gamma_{k}$ in $S^{4 n+7} / \Gamma_{k}$ is isomorphic to the complex vector bundle $\alpha$. 
We shall give a proof of the next result which can be found in [7]:

Proposition 1.8. If $i$ denotes the embedding $S^{4 n+3} / \Gamma_{k} \subset S^{4 n+7} / \Gamma_{k}$ then $i^{*} \circ(\mathrm{PD})^{-1}\left(\left[S^{4 n+3} / \Gamma_{k}, i\right]\right)=e(\alpha)$.

Proof. Denote by $\tau$ the normal bundle of $S^{4 n+3} / \Gamma_{k}$ in $S^{4 n+7} / \Gamma_{k}$ and by $h$ a classifying map: $S^{4 n+3} / \Gamma_{k} \rightarrow B U(2)$ for $\tau$. Then by 1.6 , $(\mathrm{PD})^{-1}\left(\left[S^{4 n+3} / \Gamma_{k}, i\right]\right)$ is represented by the composite:

$$
\begin{aligned}
S^{4 n+7} / \Gamma_{k} & \rightarrow\left(S^{4 n+7} / \Gamma_{k}\right) /\left(S^{4 n+7} / \Gamma_{k}-\stackrel{\circ}{N}\right) \\
& \simeq \frac{D(\tau)}{S(\tau)}=M(\tau) \stackrel{M(h)}{\longrightarrow} M U(2),
\end{aligned}
$$

$N$ being a tubular neighbourhood of $S^{4 n+3} / \Gamma_{k}$ homeomorphic to $D(\tau)$. Since the composite:

$$
\begin{aligned}
S^{4 n+3} / \Gamma_{k} & \stackrel{i}{\rightarrow} S^{4 n+7} / \Gamma_{k} \rightarrow S^{4 n+7} / \Gamma_{k} /\left(S^{4 n+7} / \Gamma_{k}-\stackrel{\circ}{N}\right) \\
& \simeq \frac{D(\tau)}{S(\tau)}=M(\tau)
\end{aligned}
$$

is the zero section: $S^{4 n+3} / \Gamma_{k} \rightarrow M(\tau)$, it follows that

$$
i^{*} \circ\left(P(D)^{-1}\right)\left(\left[S^{4 n+3} / \Gamma_{k}, i\right]\right)=e(\tau) .
$$

Since $\tau$ and $\alpha$ are isomorphic as complex vector bundles by 1.7 the proposition is proved.

In Section III we shall use the following Euler classes for $M U$ (see [6]):

$$
\begin{array}{lll}
A_{k}=e\left(\xi_{1}\right) \in \widetilde{U}^{2}\left(B \Gamma_{k}\right), & B_{k}=e\left(\xi_{2}\right) \in \widetilde{U}^{2}\left(B \Gamma_{k}\right), \\
C_{k}=e\left(\xi_{3}\right) \in \widetilde{U}^{2}\left(B \Gamma_{k}\right), & D_{k}=e\left(\eta_{1}\right) \in \widetilde{U}^{4}\left(B \Gamma_{k}\right)
\end{array}
$$

where $\xi_{1}, \xi_{2}, \xi_{3}, \eta_{1}$ are the complex vector bundles corresponding to the irreducible unitary representations $\xi_{1}: u \rightarrow 1, v \rightarrow-1, \xi_{2}: u \rightarrow$ $-1, v \rightarrow 1, \xi_{3}: k \rightarrow-1, v \rightarrow-1$ and $\eta_{1}$ as defined above.

In order to calculate $U_{*}\left(B \Gamma_{k}\right)$ we first consider the case $k=3: \Gamma_{3}=$ $\Gamma$, the quaternion group of order 8 . We recall that $\Gamma=\{ \pm 1, \pm i, \pm j, \pm k\}$ subject to the relations $i^{2}=j^{2}=k^{2}=-1, i j=k, j k=i, k i=j$. The irreducible unitary representations of $\Gamma$ are $1: i \rightarrow 1, j \rightarrow 1$, $\xi_{i}: i \rightarrow 1, j \rightarrow-1, \xi_{j}: i \rightarrow-1, j \rightarrow 1, \xi_{k}: i \rightarrow-1, j \rightarrow-1$ and $\eta: i \rightarrow\left(\begin{array}{cc}i & 0 \\ 0 & -i\end{array}\right), j \rightarrow\left(\begin{array}{cc}0 & -1 \\ 1 & 0\end{array}\right)$. The character table of $\Gamma$ is drawn on the next page.

The group $\Gamma$ acts on $S^{4 n+3}$ by means of $(n+1) \eta$ as a group of $U$ diffeomorphisms; as with $\Gamma_{k}$ we get a $U$-manifold $S^{4 n+3} / \Gamma \subset B \Gamma=$ $\bigcup_{n \geq 0} S^{4 n+3} / \Gamma$. There will be no ambiguity if we use the same notation 


\begin{tabular}{c||c|c|c|c|c|l}
\multicolumn{7}{c||}{ conjugacy classes } \\
\hline \hline 1 & 1 & -1 & $\pm i$ & $\pm j$ & $\pm k$ & \\
\hline$\xi_{i}$ & 1 & 1 & 1 & 1 & -1 & \\
\hline$\xi_{j}$ & 1 & 1 & -1 & 1 & -1 & \\
\hline$\xi_{k}$ & 1 & 1 & -1 & -1 & -1 & \\
\hline$\eta$ & 2 & 2 & 0 & 0 & 0 &
\end{tabular}

$\alpha$ as for $\Gamma_{k}$ for the complex vector bundle $S^{4 n+3} \times_{\Gamma} \mathbb{C}^{2} \rightarrow S^{4 n+3} / \Gamma$. Evidently the Propositions 1.6 and 1.7 are valid if $\Gamma_{k}$ is replaced by $\Gamma$.

In Section II the following Euler class for $M U$ will be of fundamental importance (see [6]):

$$
\begin{array}{ll}
A=e\left(\xi_{i}\right) \in \tilde{U}^{2}(B \Gamma), & B=e\left(\xi_{j}\right) \in \widetilde{U}^{2}(B \Gamma), \\
C=e\left(\xi_{k}\right) \in \widetilde{U}^{2}(B \Gamma) \quad \text { and } & D=e(\eta) \in \widetilde{U}^{4}(B \Gamma) .
\end{array}
$$

II. Calculation of $\tilde{U}_{*}(B \Gamma)$ : generators, orders and relations. The reduced homology groups $\widetilde{H}_{*}(B \Gamma)$ are such that:

$$
\widetilde{H}_{2 n}(B \Gamma)=0, \quad \widetilde{H}_{4 n+1}(B \Gamma)=\mathbb{Z}_{2} \oplus \mathbb{Z}_{2}, \quad \widetilde{H}_{4 n+3}(B \Gamma)=\mathbb{Z}_{8}, \quad n \geq 0 .
$$

The $\widetilde{U}_{*}$-AHSS of $B \Gamma$ collapses and we have a filtration of $\widetilde{U}_{n}(B \Gamma)$ :

$$
J_{-1, n+1}=0 \subset J_{0, n} \subset \cdots \subset J_{p, n-p} \subset \cdots \subset J_{n, 0}=\widetilde{U}_{n}(B \Gamma)
$$

with $J_{p, q}=\operatorname{Im}\left(\tilde{U}_{p+q}\left(X^{p}\right) \rightarrow \widetilde{U}_{p+q}(B \Gamma)\right), X^{p}$ being the $p$-skeleton of $B \Gamma$. Moreover $J_{p, q} / J_{p-1, q+1}=\widetilde{H}_{p}\left(B \Gamma, U_{q}(p t)\right)$.

Proposition 2.1. (a) $\widetilde{U}_{2 n}(B \Gamma)=0, \widetilde{U}_{2 n+1}(B \Gamma)=U_{2 n+1}(B \Gamma)$, $U_{2 n}(B \Gamma)=U_{2 n}(p t)$.

(b) $\operatorname{Ord}\left(\widetilde{U}_{4 n+3}(B \Gamma)\right)=2^{r}$,

$$
\begin{aligned}
r= & 3\left(\sum_{i=0}^{n} \operatorname{Rank} U_{4 i}(p t)\right) \\
& +2\left(\sum_{i=0}^{n} \operatorname{Rank} U_{4 i+2}(p t)\right) ; \quad \operatorname{Ord}\left(\widetilde{U}_{4 n+1}(B \Gamma)\right)=2^{s},
\end{aligned}
$$




$$
s=3\left(\sum_{i=0}^{n-1} \operatorname{Rank} U_{4 i+2}(p t)\right)+2\left(\sum_{i=0}^{n} \operatorname{Rank} U_{4 i}(p t)\right) .
$$

Proof. (a) From the filtration $J_{-1,2 n+1}=0 \subset J_{0,2 n} \subset \cdots \subset J_{p, 2 n-p} \subset$ $\cdots \subset J_{2 n, 0}$, and $J_{p, 2 n-p} / J_{p-1,2 n-p+1}=H_{p}\left(B \Gamma, U_{2 n-p}(p t)\right)=0$ it follows that $\widetilde{U}_{2 n}(B \Gamma)=0$. Hence $U_{2 n}(B \Gamma)=U_{2 n}(p t)$ and $\widetilde{U}_{2 n+1}(B \Gamma)=$ $U_{2 n+1}(B \Gamma)$ because $U_{2 n+1}(p t)=0$.

(b) The orders are easy consequences of:

$$
\begin{gathered}
J_{4 p+3,2 q} / J_{4 p+2,2 q+1}=H_{4 p+3}\left(B \Gamma, U_{2 q}(p t)\right) \\
=\mathbb{Z}_{8} \otimes U_{2 q}(p t)=U_{2 q}(p t) / 8 . U_{2 q}(p t), \\
J_{4 p+2,2 q+1} / J_{4 p+1,2 q+2}=0 \\
J_{4 p+1,2 q+2} / J_{4 p, 2 q+3} \\
=U_{2 q+2}(p t) / 2 U_{2 q+2}(p t) \oplus U_{2 q+2}(p t) / 2 U_{2 q+2}(p t), \\
J_{4 p, 2 q+3} / J_{4 p-1,2 q+4}=0 .
\end{gathered}
$$

Let $w_{4 n+3} \in \widetilde{U}_{4 n+3}(B \Gamma)$ be $\left[S^{4 n+3} / \Gamma, q\right], q$ being the inclusion $S^{4 n+3} / \Gamma \subset B \Gamma, u_{4 n+1}=A \cap w_{4 n+3} \in \widetilde{U}_{4 n+1}(B \Gamma), v_{4 n+1}=B \cap w_{4 n+3} \in$ $\widetilde{U}_{4 n+1}(B \Gamma)$.

TheOREM 2.2. The set $\left\{u_{4 n+1}, v_{4 n+1}, w_{4 n+3}\right\}_{n \geq 0}$ is a system of generators for the $U_{*}(p t)$-module $\widetilde{U}_{*}(B \Gamma)$.

Proof. Since the $U_{*}$-AHSS for $B \Gamma$ collapses we can use 1.1. If $\mu^{\prime}$ denotes the edge homomorphism it is enough to prove that $\mu^{\prime}\left(w_{4 n+3}\right)$, $\left\{\mu^{\prime}\left(u_{4 n+1}\right), \mu^{\prime}\left(v_{4 n+1}\right)\right\}$ are systems of generators respectively for $\widetilde{H}_{4 n+3}(B \Gamma)$ and $\widetilde{H}_{4 n+1}(B \Gamma)$.

(a) Consider the following commutative diagram:

$$
\begin{array}{ccc}
\widetilde{U}_{4 n+3}\left(S^{4 n+3} / \Gamma\right) \stackrel{q_{*}}{\longrightarrow} & \widetilde{U}_{4 n+3}(B \Gamma) \\
\mu^{\prime} \downarrow & \downarrow^{\prime} \\
\widetilde{H}_{4 n+3}\left(S^{4 n+3} / \Gamma\right) \stackrel{q_{*}}{\longrightarrow} \widetilde{H}_{4 n+3}(B \Gamma) .
\end{array}
$$

We have $\mu^{\prime}\left(\left[S^{4 n+3} / \Gamma, 1\right]\right)=c_{1}\left(S^{4 n+3} / \Gamma\right)$, where $c_{1}\left(S^{4 n+3} / \Gamma\right)$ denotes the fundamental class of $S^{4 n+3} / \Gamma$ (for $H$ ). Since $c_{1}\left(S^{4 n+3} / \Gamma\right)$ is a generator of $\widetilde{H}_{4 n+3}(B \Gamma)$ it follows that $q_{*}\left(c_{1}\left(S^{4 n+3} / \Gamma\right)\right)$ is a generator of $\widetilde{H}_{4 n+3}(B \Gamma)$ because $S^{4 n+3} / \Gamma$ is the $(4 n+3)$-skeleton of $B \Gamma$. Now $q_{*}\left(\left[S^{4 n+3} / \Gamma, 1\right]\right)=\left[S^{4 n+3} / \Gamma, q\right]$ and then $\mu^{\prime}\left(\left[S^{4 n+3} / \Gamma, q\right]\right)$ is a generator of $\widetilde{H}_{4 n+3}(B \Gamma)$. 
(b) By [6], Section II, $\mu(A)$ and $\mu(B)$ generate the group $H^{2}(B \Gamma)$ and then if $A_{1}=q^{*}(A) \in U^{2}\left(S^{4 n+3} / \Gamma\right), B_{1}=q^{*}(B) \in U^{2}\left(S^{4 n+3} / \Gamma\right)$, then the elements $\mu\left(A_{1}\right), \mu\left(B_{1}\right)$ generate $H^{2}\left(S^{4 n+3} / \Gamma\right)$ because the following diagram commutes:

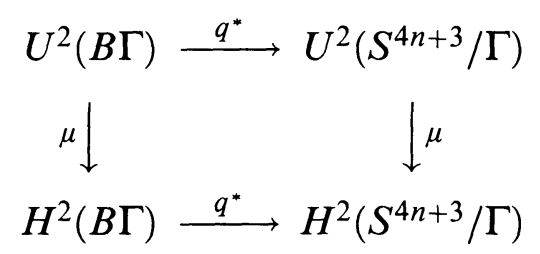

and the bottom line is an isomorphism. Consider $t_{4 n+3}=\left[S^{4 n+3} / \Gamma, 1\right]$ $\in U_{4 n+3}\left(S^{4 n+3} / \Gamma\right)$; then $\mu^{\prime}\left(t_{4 n+3}\right)=c_{1}\left(S^{4 n+3} / \Gamma\right)$. Since the diagram:

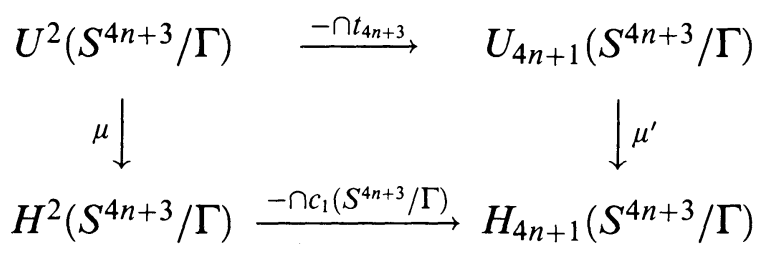

commutes by 1.5 and since the bottom line is an isomorphism it follows that $\mu^{\prime}\left(A_{1} \cap t_{4 n+3}\right)$ and $\mu^{\prime}\left(B_{1} \cap t_{4 n+3}\right)$ generate the group $H_{4 n+1}\left(S^{4 n+3} / \Gamma\right)$. Now by using the commutative diagram:

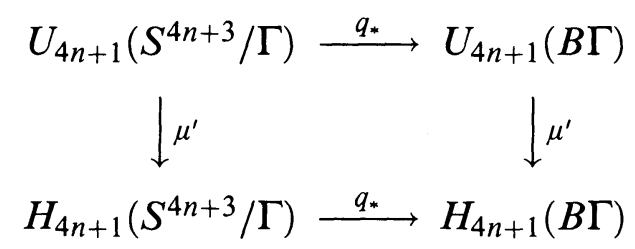

we see that $q_{*}\left(A_{1} \cap t_{4 n+3}\right)$ and $q_{*}\left(B_{1} \cap t_{4 n+3}\right)$ generate the group $H_{4 n+1}(B \Gamma)$. Since $q_{*}\left(A_{1} \cap t_{4 n+3}\right)=q_{*}\left(q^{*}(A) \cap t_{4 n+3}\right)=A \cap q_{*}\left(t_{4 n+3}\right)=$ $A \cap w_{4 n+3}$ and $q_{*}\left(B_{1} \cap t_{4 n+3}\right)=B \cap w_{4 n+3}$ the assertion (b) has been proved.

(1) Relations between the generators. We first recall the definition of the pull back transfer. Let $M^{n}$ be a closed $U$-manifold, $N^{m}$ a closed $U$-submanifold of $M^{n}$ with $(n-m)$ even and $i$ the inclusion $N^{m} \subset M^{n}$. If $\left[V^{r}, f\right] \in U_{r}\left(M^{n}\right)$, then there is a weakly complex representative map $g: V^{r} \rightarrow M^{n}$ transversal to $N^{m}$. Hence $g^{-1}\left(N^{m}\right)$ is a smooth closed submanifold of $V^{r}$ and $\operatorname{dim} g^{-1}\left(N^{m}\right)=r+m-$ $n$. Since $N^{m}$ is a $U$-submanifold of $M^{n}$ the normal vector bundle $\tau$ of $N^{m}$ is in fact a complex vector bundle and by transversality we have $T\left(W^{r+m-n}\right)+g_{1}^{*}(\tau)=j^{*}\left(T\left(V^{r}\right)\right)(1)$ where $W^{r+m-n}=g^{-1}\left(N^{m}\right)$, $g_{1}=g \mid g^{-1}\left(N^{m}\right), j: W^{r+m-n} \subset V^{r}$ and $T(-)$ being the tangent vector 
bundle. Since $V^{r}$ is a $U$-manifold the stable tangent bundle of $V^{r}$ has a complex structure and the above relation (1) determines a unique complex structure on the stable tangent bundle of $W^{r+m-n}$ (see [5], page 16). Then we define $i !: U_{r}\left(M^{n}\right) \rightarrow U_{r+m-n}\left(N^{m}\right)$ by $i !\left(\left[V^{r}, f\right]\right)=$ $\left[W^{r+m-n}, g_{1}\right]$. Moreover, the following diagram is commutative:

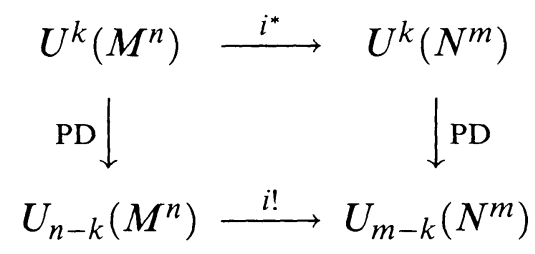

PD being the Poincaré duality (see [2], [7]).

Now, there is a map $\Delta: \widetilde{U}_{*}(B \Gamma) \rightarrow \widetilde{U}_{*}(B \Gamma)$ defined by $\Delta(x)=D \cap x$, with $D=e(\eta)$, the Euler class of $\eta$. The map $\Delta$ is a homomorphism of graded $U_{*}(p t)$-modules of degree -4 .

Proposition 2.3. We have

$$
\begin{gathered}
\Delta\left(w_{4 n+3}\right)=w_{4(n-1)+3}, \quad \Delta\left(u_{4 n+1}\right)=u_{4(n-1)+1}, \\
\Delta\left(v_{4 n+1}\right)=v_{4(n-1)+1}, \quad n \geq 0 .
\end{gathered}
$$

Proof. Let $p, r, s$ be respectively the inclusions $S^{4(n-1)+3} / \Gamma \subset$ $S^{4 n+3} / \Gamma, S^{4 n+3} / \Gamma \subset S^{4 n+7} / \Gamma, S^{4 n+7} / \Gamma \subset B \Gamma$. Then

$$
\left[S^{4 n+3} / \Gamma, r\right] \in U_{4 n+3}\left(S^{4 n+7} / \Gamma\right) .
$$

We have the pull back transfer

$$
r !: U_{4 n+3}\left(S^{4 n+7} / \Gamma\right) \rightarrow U_{4(n-1)+3}\left(S^{4 n+3} / \Gamma\right)
$$

and the commutative diagram:

$$
\begin{array}{ccc}
U^{4}\left(S^{4 n+7} / \Gamma\right) & \stackrel{r^{*}}{\longrightarrow} & U^{4}\left(S^{4 n+3} / \Gamma\right) \\
\mathrm{PD} \downarrow & & \downarrow \mathrm{PD} \\
U_{4 n+3}\left(S^{4 n+7} / \Gamma\right) \stackrel{r !}{\longrightarrow} & U_{4(n-1)+3}\left(S^{4 n+3} / \Gamma\right) .
\end{array}
$$

The element $r !\left(\left[S^{4 n+3} / \Gamma, i\right]\right)$ is $\left[g^{-1}\left(S^{4 n+3} / \Gamma\right), g \mid g^{-1}\left(S^{4 n+3} / \Gamma\right)\right]$ where $g$ is the map: $S^{4 n+3} / \Gamma \rightarrow S^{4 n+7} / \Gamma$ defined by $g\left(\left[z_{1}, z_{2}, \ldots, z_{2 n+2}\right]\right)=$ $\left[z_{1}, z_{2}, \ldots, z_{2 n}, 0,0, z_{2 n+2}\right]$ because $g$ is homotopic to $r$ and transversal to $S^{4 n+3} / \Gamma$. But $g^{-1}\left(S^{4 n+3} / \Gamma\right)=S^{4(n-1)+3} / \Gamma$ and $g \mid g^{-1}\left(S^{4 n+3} / \Gamma\right)=$ p. It is easily seen that

$$
r !\left(\left[S^{4 n+3} / \Gamma, r\right]\right)=\left[S^{4(n-1)+3} / \Gamma, p\right] \in U_{4(n-1)+3}\left(S^{4 n+3} / \Gamma\right),
$$


the $U$-structure on $S^{4(n-1)+3} / \Gamma$ being the canonical one (this result can be found in [7], Lemma 2.5, page 145). Now by 1.8 we have $r^{*} \circ$ $(P D)^{-1}\left(\left[S^{4 n+3} / \Gamma, r\right]\right)=e(\alpha), \alpha$ being $\mathbb{C}$-vector bundle $S^{4 n+3} \times_{\Gamma} \mathbb{C}^{2} \rightarrow$ $S^{4 n+3} / \Gamma, \Gamma$ acting on $S^{4 n+3}$ and $\mathbb{C}^{2}$ respectively by using $(n+1) \eta$ and $\eta$ (see Section I). Since $\alpha=(s \circ r)^{*}(\eta)\left(\eta: E \times_{\Gamma} \mathbb{C}^{2} \rightarrow B \Gamma\right)$, we have $e(\alpha)=$ $(s \circ r)^{*}(D)$ and then $r^{*} \circ(\mathrm{PD})^{-1}\left(\left[S^{4 n+3} / \Gamma, r\right]\right)=(s \circ r)^{*}(D)$. From the above diagram it follows that $(s \circ r)^{*}(D)=(\mathrm{PD})^{-1}\left(\left[S^{4(n-1)+3} / \Gamma, p\right]\right)$. The fundamental class of $S^{4 n+3} / \Gamma$ for $M U$ involved in the Poincaré duality being $\left[S^{4 n+3} / \Gamma, 1\right] \in U_{4 n+3}\left(S^{4 n+3} / \Gamma\right)$ (see 1.3) we have:

$$
(s \circ r)^{*}(D) \cap\left[S^{4 n+3} / \Gamma, 1\right]=\left[S^{4(n-1)+3} / \Gamma, p\right]
$$

and consequently

$$
\begin{aligned}
w_{4(n-1)+3} & =(s \circ r)_{*}\left(\left[S^{4(n-1)+3} / \Gamma, p\right]\right) \\
& =(s \circ r)_{*}\left[(s \circ r)^{*}(D) \cap\left[S^{4 n+3} / \Gamma, 1\right]\right] \\
& =D \cap(s \circ r)_{*}\left(\left[S^{4 n+3} / \Gamma, 1\right]\right) \\
& =D \cap\left[S^{4 n+3} / \Gamma, s \circ r\right]=D \cap w_{4 n+3}=\Delta\left(w_{3 n+3}\right) .
\end{aligned}
$$

We have

$$
\begin{aligned}
\Delta\left(u_{4 n+1}\right) & =\Delta\left(A \cap w_{4 n+3}\right)=(D \cdot A) \cap\left(w_{4 n+3}\right) \\
& =A \cap\left[D \cap w_{4 n+3}\right]=A \cap w_{(n-1)+3}=u_{4(n-1)+1}
\end{aligned}
$$

Similarly $\Delta\left(v_{4 n-1}\right)=v_{4(n-1)+1}$.

REMARK. The homomorphism $\Delta$ is sometimes called the Smithhomomorphism.

We recall from [6], Lemma 2.11 and Theorem 2.12, that if $\Lambda_{*}$ denotes the $U^{*}(p t)$-graded algebra $U^{*}(p t)[[X, Y, Z]], \operatorname{dim} X=\operatorname{dim} Y=$ $2, \operatorname{dim} Z=4$ and $\Omega_{*}$ the sub- $U^{*}(p t)$-algebra $U^{*}(p t)[[Z]]$ then there is $T(Z)=8 Z+2 \lambda_{2} Z^{2}+\sum_{i \geq 3} \lambda_{i} Z^{i} \in \Omega_{4}, \lambda_{2} \notin 2 U^{*}(p t)$, such that: $M(D)=0\left(M(Z) \in \Omega_{*}\right)$ iff $M(Z) \in T(Z) \Omega_{*}$. Moreover by [6], Lemmas $2.13,2.15$, there is

$$
J(Z)=\mu_{1} Z+\sum_{i \geq 2} \mu_{i} Z^{i} \in \Omega_{0}, \quad \mu_{1} \notin 2 U^{*}(p t),
$$

such that: $E(D)+A M(D)+B N(D)=0$ iff $M(Z), N(Z)$ belong to $(2+J(Z)) \Omega_{*}$ and $E(Z)$ to $T(Z) \Omega_{*}(M(Z), N(Z), E(Z)$ are elements of $\Omega_{*}$ ). We also recall the following notation: if $M(Z)=$ $\sum_{i \geq r} a_{i} Z^{i} \in \Omega_{2 n}$ with $a_{r} \neq 0$ then $\nu(M)=4 r$. Let $W, V_{1}, V_{2}$ be the $U_{*}(p t)$-submodules of $\widetilde{U}_{*}(B \Gamma)$ generated respectively by $\left\{W_{4 n+3}\right\}_{n \geq 0}$, $\left\{u_{4 n+1}\right\}_{n \geq 0},\left\{v_{4 n+1}\right\}_{n \geq 0}$. 
THEOREM 2.4. (a) $\widetilde{U}_{*}(B \Gamma)=W \oplus V_{1} \oplus V_{2}$.

(b) In $\widetilde{U}_{2 p+1}(B \Gamma)$ we have $0=a_{0} w_{3}+a_{1} w_{7}+\cdots+a_{n} w_{4 n+3}=b_{0} u_{1}+$ $\cdots+b_{m} u_{4 m+1}$ iff there are homogeneous polynomials $M(Z), M_{1}(Z)$ and homogeneous formal power series $N(Z), N_{1}(Z)$ of $\Omega_{*}$ satisfying. $b_{m} Z+b_{m-1} Z^{2}+\cdots+b_{0} Z^{m+1}=M(Z)(2+J(Z))+N(Z), a_{n} Z+$ $a_{n-1} Z^{2}+\cdots+a_{0} Z^{n+1}=M_{1}(Z) T(Z)+N_{1}(Z), \nu(N)>4(m+1)$, $\nu\left(N_{1}\right)>4(n+1)$. Moreover: $b_{0} u_{1}+\cdots+b_{m} u_{4 m+1}=0$ iff $b_{0} v_{1}+\cdots+$ $b_{m} v_{4 m+1}=0$.

Proof. (a) Suppose that $\left(a_{0} w_{3}+\cdots+a_{n} w_{4 n+3}\right)+\left(b_{0} u_{1}+\cdots+\right.$ $\left.b_{m} u_{4 m+1}\right)+\left(c_{0} v_{1}+\cdots+c_{r} v_{4 r+1}\right)=0$. Then a proof similar to that of Lemma 2.14 of [6] shows that $b_{m}=2 d_{m}, d_{m} \in U_{*}(p t)$. Consider $H(Z)=b_{m} Z+b_{m-1} Z^{2}+\cdots+b_{0} Z^{m+1}$; we have: $H(Z)-$ $d_{m} Z(2+J(Z))=b_{m-1}^{\prime} Z^{2}+\cdots+b_{0}^{\prime} Z^{m+1}+F(Z), \nu(F)>4(m+1)$. Then $A H(D)=A\left[b_{m-1}^{\prime} D^{2}+\cdots+b_{0}^{\prime} D^{m+1}\right]+A F(D)$ and by taking the cup product by $w_{4 m+7}$ we obtain $b_{0} u_{1}+\cdots+b_{m} u_{4 m+1}=$ $b_{0}^{\prime} u_{1}+\cdots+b_{m-1}^{\prime} u_{4(m-1)+1}$. As seen before, we have: $b_{m-1}^{\prime}=2 d_{m-1}^{\prime}$, $d_{m-1}^{\prime} \in U_{*}(p t)$. We repeat the same process and after a finite number of operations we get $b_{m} Z+\cdots+b_{0} Z^{m+1}=M(Z)(2+J(Z))+$ $N(Z), M(Z)$ being a homogeneous polynomial and $N(Z)$ a homogeneous formal power series such that $\nu(N)>4(m+1)$. Hence $b_{0} u_{1}+\cdots+b_{m} u_{4 m+1}=M(D) A(2+J(D)) \cap w_{4 m+7}=0$. Similarly $c_{0} v_{1}+\cdots+c_{r} v_{4 r+1}=0$ which ends the proof of part (a).

(b) Suppose that $a_{0} w_{3}+\cdots+a_{n} w_{4 n+3}=0$. As in Proposition 2.6 of [6] we have $a_{n}=8 e_{n}, e_{n} \in U_{*}(p t)$. We form $a_{n} Z+\cdots+a_{0} Z^{n+1}-$ $e_{n} T(Z)=a_{n-1}^{\prime} Z^{2}+\cdots+a_{0}^{\prime} Z^{n+1}+F_{1}(Z), \nu\left(F_{1}\right)>4(n+1)$ and by taking the cup-product by $w_{4 n+7}$ we obtain: $a_{0} w_{3}+\cdots+a_{n} w_{4 n+3}=$ $a_{0}^{\prime} w_{3}+a_{n-1}^{\prime} w_{4(n-1)+3}$. As before, we have $a_{n-1}^{\prime}=8 e_{n-1}^{\prime}, e_{n-1}^{\prime} \in U_{*}(p t)$. We repeat the same process with $a_{n-1}^{\prime} Z^{2}+a_{n-2}^{\prime} Z^{3}+\cdots+a_{0}^{\prime} Z^{n+1}$ and after a finite number of operations we get: $a_{n} Z+\cdots+a_{0} Z^{n+1}=$ $M_{1}(Z) T(Z)+N_{1}(Z), \nu\left(N_{1}\right)>4(n+1), M_{1}(Z)$ being a homogeneous polynomial and $N_{1}(Z)$ a homogeneous formal power series. The proof of part (a) shows that $b_{m} Z+\cdots+b_{0} Z^{n+1}=M(Z)(2+J(Z))+N(Z)$, $\nu(N)>4(m+1)$. The remaining part of $(\mathrm{b})$ is evident.

(2) Orders of the Generators.

LEMMA 2.5. Suppose $t \in \widetilde{U}_{2 n+1}(B \Gamma), t \neq 0$. If $a \in U_{4}(p t)$ is such that $a \notin 2 U_{4}(p t)$ then $a \cdot t \neq 0$.

Proof. Since $t \neq 0$ there is an integer $q \geq 0$ such that $t \in J_{q, 2 n+1-q}$ and $t \notin J_{q-1,2 n+1-(q-1)}$. We have either $q=4 s+3$ or $q=4 s+1$. 
Suppose $q=4 s+3$. We have the following commutative diagram:

$$
\begin{array}{ccc}
U_{4}(p t) \otimes J_{4 s+3,2 n+1-(4 s+3)} & \chi & J_{4 s+3,2(n-2 s+1)} \\
1 \otimes h \downarrow & \downarrow h \\
U_{4}(p t) \otimes U_{2(n-2 s-1)}(p t) \otimes \mathbb{Z}_{8} & \stackrel{x \otimes 1}{\longrightarrow} U_{2(n-2 s+1)} \otimes \mathbb{Z}_{8}
\end{array}
$$

where $h$ is the canonical map: $J_{* *} \rightarrow E_{* *}^{\infty}=H_{*}\left(B \Gamma, U_{*}(p t)\right)=U_{*}(p t) \otimes$ $H_{*}(B \Gamma)$. It is enough to prove that in $U_{*}(p t)=\mathbb{Z}\left[x_{1}, x_{2}, \ldots, x_{4}, \ldots\right]$ if $a \in U_{4}(p t), a \notin 2 U_{4}(p t), b \in U_{2 k}(p t), b \notin 8 U_{2 k}(p t)$ then $a b \notin$ $8 U_{2(k+2)}(p t)$; we may suppose that $a$ and $b$ are monomials and then the assertion is clear. The case $q=4 s+1$ is similar.

THEOREM 2.6. We have ord $w_{4 n+3}=2^{2 n+3}$.

Proof. (a) ord $w_{3}=2^{3}$.

We have $0=T(D)=2^{3} D+H(D) D^{2}$ and $0=T(D) \cap w_{7}=2^{2} w_{3}+$ $H(D) \cap\left(D^{2} \cap w_{7}\right)=2^{3} w_{3}$ because $D^{2} \cap w_{7} \in U_{-1}(B \Gamma)=0$. Then by using the edge homomorphism $\mu^{\prime}: \widetilde{U}_{3}(B \Gamma) \rightarrow \widetilde{H}_{3}(B \Gamma)=\mathbb{Z}_{8}$ we see that $2^{2} w_{3} \neq 0$. Hence ord $w_{3}=2^{3}$.

(b) Suppose ord $w_{4 i+3}=2^{2 i+3}, 0 \leq i \leq n-1$.

We have $0=T(D)=2^{3} D+2 \lambda_{2} D^{2}+\lambda_{3} D^{3}+\cdots+\lambda_{n+1} D^{n+1}+H(D) D^{n+2}$, $\lambda_{2} \in U^{-4}(p t)=U_{4}(p t), \lambda_{2} \notin 2 U_{4}(p t)$. Take the cup-product by $w_{4 n+7}: 2^{3} w_{4 n+3}+2 \lambda_{2} w_{4(n-1)+3}+\lambda_{3} w_{4(n-2)+3}+\cdots+\lambda_{n+1} w_{3}=0$ and after multiplication by $2^{2 n-1}$ we get: $2^{2 n+2} w_{4 n+3}+\lambda_{2} 2^{2 n} w_{4(n-1)+3}=0$; since ord $w_{4(n-1)+3}=2^{2 n+1}$ we have $2^{2 n} w_{4(n-1)+3} \neq 0$ and by 2.5 $\lambda_{2} 2^{2 n} w_{4(n-1)+3} \neq 0$ because $\lambda_{2} \notin 2 U_{4}(p t)$. Hence $2^{2 n+2} w_{4 n+3} \neq 0$. Now we have: $2^{2 n+3} w_{4 n+3}=-\lambda_{2} 2^{2 n+1} w_{4(n-1)+3}=0$. It follows that ord $w_{4 n+3}=2^{2 n+3}$ which ends the proof of 2.6 .

LEMMA 2.7. If $G_{n}=U_{4 n-2}(p t) w_{3}+U_{4 n}(p t) u_{1}+U_{4 n}(p t) v_{1}, G_{n}^{\prime}=$ $U_{4 n}(p t) w_{3}+U_{4 n+2}(p t) u_{1}+U_{4 n+2}(p t) v_{1}$ then we have the exact sequences:

$$
\begin{aligned}
& 0 \rightarrow G_{n} \rightarrow \widetilde{U}_{4 n+1}(B \Gamma) \stackrel{\Delta}{\rightarrow} \widetilde{U}_{4(n-1)+1}(B \Gamma) \rightarrow 0 \\
& 0 \rightarrow G_{n}^{\prime} \rightarrow \widetilde{U}_{4 n+3}(B \Gamma) \stackrel{\Delta}{\rightarrow} \widetilde{U}_{4(n-1)+3}(B \Gamma) \rightarrow 0
\end{aligned}
$$

Proof. We wish to show that the sequence:

$$
0 \rightarrow G_{n} \rightarrow \widetilde{U}_{4 n+1}(B \Gamma) \stackrel{\Delta}{\rightarrow} \widetilde{U}_{4(n-1)+1}(B \Gamma) \rightarrow 0
$$


is exact. It follows by 2.3 that $\Delta$ is surjective and $G_{n} \subset \operatorname{ker} \Delta$. Suppose $0=a w_{3}+b u_{1}+c v_{1}, a \in U_{4 n-2}(p t), b \in U_{4 n}(p t), c \in U_{4 n}(p t)$. Then $a \cdot w_{3} \in J_{3,4 n-2}$ and since $b u_{1}+c v_{1} \in J_{1,4 n}$ we have a $w_{3} \in J_{2,4 n-1} \supset$ $J_{1,4 n}$. If $h$ denotes the quotient map: $J_{3,4 n-2} \rightarrow J_{3,4 n-2} / J_{2,4 n-1}=$ $H^{3}\left(B \Gamma, U_{4 n-2}(p t)\right)=U_{4 n-2}(p t) / 8 U_{4 n-2}(p t)$, it follows that $h\left(a w_{3}\right)=$ 0 and consequently $a=2^{3} a^{\prime}$. Hence $a w_{3}=a^{\prime} 2^{3} w_{3}=0$ and then $b u_{1}+c v_{1}=0$. Similarly we have $b=2 b^{\prime}, c=2 c^{\prime}$ which means that $0=a w_{3}+b u_{1}+c v_{1}\left(a \in U_{4 n-2}(p t), b \in U_{4 n}(p t), c \in U_{4 n}(p t)\right)$ if and only if $a=2^{3} a^{\prime}, b=2 b^{\prime}, c=2 c^{\prime}$. Hence ord $G_{n}=2^{k}$, $k=3 \operatorname{Rank} U_{4 n-2}(p t)+2 \operatorname{Rank} U_{4 n}(p t)$. Now, we have ord ker $\Delta=$ ord $\widetilde{U}_{4 n+1}(B \Gamma) /$ ord $\widetilde{U}_{4(n-1)+1}(B \Gamma)=2^{k}$ by 2.1 . From $G_{n} \subset \operatorname{ker} \Delta$ and ord $G_{n}=$ ord $\operatorname{ker} \Delta$ we see that the sequence $0 \rightarrow G_{n} \rightarrow \widetilde{U}_{4 n+1}(B \Gamma) \stackrel{\Delta}{\rightarrow}$ $\widetilde{U}_{4(n-1)+1}(B \Gamma) \rightarrow 0$ is exact. A similar proof shows that the sequence $0 \rightarrow G_{n}^{\prime} \rightarrow \widetilde{U}_{4 n+3}(B \Gamma) \stackrel{\Delta}{\rightarrow} \widetilde{U}_{4(n-1)+3}(B \Gamma) \rightarrow 0$ is exact.

THEOREM 2.8. We have ord $u_{4 n+1}=$ ord $v_{4 n+1}=2^{n+1}$.

Proof. If $n=0$ the assertion is clear. Suppose ord $u_{4 i+1}=2^{i+1}$, $0 \leq i \leq n-1$. Then $\Delta\left(2^{n} u_{4 n+1}\right)=2^{n} u_{4(n-1)+1}=0$ and since the sequence $0 \rightarrow G_{n} \rightarrow U_{4 n+1}(B \Gamma) \stackrel{\Delta}{\rightarrow} U_{4(n-1)+1}(B \Gamma) \rightarrow 0$ is exact, (see 2.7), there are $a \in U_{4 n-2}(p t), b \in U_{4 n}(p t), c \in U_{4 n}(p t)$ such that $2^{n} u_{4 n+1}=a w_{3}+b u_{1}+c v_{1}$. It follows that $-b u_{1}+2^{n} \cdot u_{4 n+1}=0$ and $2^{n+1} \cdot u_{4 n+1}=0$; hence ord $u_{4 n+1} \leq 2^{n+1}$. By Theorem 2.4 there are $M(Z), N(Z)$ in $\Omega_{*}$ such that: $2^{n} Z-b Z^{n+1}=M(Z)(2+J(Z))+N(Z)$, $\nu(N)>4(n+1)$. If $M(Z)=h_{1} Z+h_{2} Z^{2}+\cdots$, then we have:

$$
\begin{aligned}
2^{n} Z-b Z^{n+1}= & \left(2+\mu_{1} Z+\mu_{2} Z^{2}+\cdots\right)\left(h_{1} Z+h_{2} Z^{2}+\cdots\right) \\
& +e_{n+2} Z^{n+2}+e_{n+3} Z^{n+3}+\cdots, \quad \mu_{1} \notin 2 U_{*}(p t) .
\end{aligned}
$$

A straightforward calculation shows that $2^{n-j} \mid h_{j}$ and $2^{n-j+1} \nmid h_{j}, 1 \leq$ $j \leq n$. We have: $-b=2 h_{n+1}+\mu_{1} h_{n}+\mu_{2} h_{n-1}+\cdots+\mu_{n} h_{1}$; as $2 \mid h_{j}$, $1 \leq j \leq n-1,2 \nmid h_{n}, 2 \nmid \mu_{1}$ we have $2 \nmid b$. As a consequence we get $2^{n} u_{4 n+1} \neq 0$ and ord $u_{4 n+1}=2^{n+1}$. Similarly ord $v_{4 n+1}=2^{n+1}$.

III. $\widetilde{U}^{*}\left(B \Gamma_{k}\right), k \geq 4$ : generators, orders and relations. We have seen in [6], Section III, that there are elements $D_{k} \in \widetilde{U}^{4}\left(B \Gamma_{k}\right), B_{k} \in$ $\widetilde{U}^{2}\left(B \Gamma_{k}\right), C_{k} \in \widetilde{U}^{2}\left(B \Gamma_{k}\right)$ defined as Euler classes of irreducible unitary representations $\eta_{1}, \xi_{2}, \xi_{3}$ of $\Gamma_{k}$. Moreover in the same article 
(Sec. III) we have determined three homogeneous formal power series $T_{k}(Z) \in \Omega_{4}, J_{k}(Z) \in \Omega_{0}, G_{k}(Z) \in \Omega_{2}$ such that $B_{k}\left(2+J\left(D_{k}\right)\right)+$ $G_{k}\left(D_{k}\right)=C_{k}\left(2+J\left(D_{k}\right)\right)+G_{k}\left(D_{k}\right)=0$ and there is $G_{k}^{\prime}(Z) \in \Omega_{2}$ satisfying $G_{k}(Z)=(2+J(Z)) G_{k}^{\prime}(Z)$. Then with $B_{k}^{\prime}=B_{k}+G_{k}^{\prime}\left(D_{k}\right)$, $C_{k}^{\prime}=C_{k}+G_{k}^{\prime}\left(D_{k}\right)$ and $\mu$ being the edge homomorphism: $U^{2}\left(B \Gamma_{k}\right) \rightarrow$ $H^{2}\left(B \Gamma_{k}\right)$ we see that $\mu\left(B_{k}^{\prime}\right)=\mu\left(B_{k}\right)$ and $\mu\left(C_{k}^{\prime}\right)=\mu\left(C_{k}\right)$ are generators of the group $H^{2}\left(B \Gamma_{k}\right) ; \mu\left(D_{k}\right)$ is obviously a generator of $H^{4}\left(B \Gamma_{k}\right)$. Moreover $B_{k}^{\prime}\left(2+J\left(D_{k}\right)\right)=C_{k}^{\prime}\left(2+J\left(D_{k}\right)\right)=0$.

Now let $w_{4 n+3}^{\prime} \in \widetilde{U}_{4 n+3}\left(B \Gamma_{k}\right)$ be $\left[S^{4 n+3} / \Gamma_{k}, q^{\prime}\right], q^{\prime}$ being the inclusion $S^{4 n+3} / \Gamma_{k} \subset B \Gamma_{k}, u_{4 n+1}^{\prime}=B_{k}^{\prime} \cap w_{4 n+3}^{\prime} \in \widetilde{U}_{4 n+1}, v_{4 n+1}^{\prime}=$ $C_{k}^{\prime} \cap w_{4 n+1}^{\prime} \in \widetilde{U}_{4 n+1}\left(B \Gamma_{k}\right)$. Then we have the following theorems whose proofs are identical respectively to Theorem 2.2 and Theorem 2.4 and therefore will be omitted.

THEOREM 3.1. The set $\left\{u_{4 n+1}^{\prime}, v_{4 n+1}^{\prime}, w_{4 n+3}^{\prime}\right\}_{n \geq 0}$ is a system of generators for the $U(p t)$-module $\widetilde{U}_{*}\left(B \Gamma_{k}\right)$.

Now let $W^{\prime}, V_{1}^{\prime}, V_{2}^{\prime}$ be the $U_{*}(p t)$-submodules of $\widetilde{U}_{*}\left(B \Gamma_{k}\right)$ generated respectively by $\left\{w_{4 n+3}^{\prime}\right\}_{n \geq 0},\left\{u_{4 n+1}^{\prime}\right\}_{n \geq 0},\left\{v_{4 n+1}^{\prime}\right\}_{n \geq 0}$.

THEOREM 3.2. (a) $\widetilde{U}_{*}\left(B \Gamma_{k}\right)=W^{\prime} \oplus V_{1}^{\prime} \oplus V_{2}^{\prime}$.

(b) In $\widetilde{U}_{2 p+1}\left(B \Gamma_{k}\right)$ we have $0=a_{0} w_{3}^{\prime}+a_{1} w_{7}^{\prime}+\cdots+a_{n} w_{4 n+3}^{\prime}=b_{0} u_{1}^{\prime}+$ $\cdots+b_{m} u_{4 m+1}^{\prime}$ iff there are homogeneous polynomials $M(Z), M_{1}(Z)$ and homogeneous formal power series $N(Z), N_{1}(Z)$ of $\Omega_{*}$ satisfying: $b_{m} Z+b_{m-1} Z^{2}+\cdots+b_{0} Z^{m+1}=M(Z)(2+J(Z))+N(Z), a_{n} Z+$ $a_{n-1} Z^{2}+\cdots+a_{0} Z^{n+1}=M_{1}(Z) T_{k}(Z)+N_{1}(Z), \nu(N)>4(m+1)$, $\nu\left(N_{1}\right)>4(n+1)$. Moreover $b_{0} u_{1}^{\prime}+\cdots+b_{m} u_{4 m+1}^{\prime}=0$ iff $b_{0} v_{1}^{\prime}+\cdots+$ $b_{m} v_{4 m+1}^{\prime}=0$.

There is a Smith homomorphism $\Delta: \widetilde{U}_{*}\left(B \Gamma_{k}\right) \rightarrow \widetilde{U}_{*}\left(B \Gamma_{k}\right)$ of degree -4 such that

$$
\begin{aligned}
\Delta\left(w_{4 n+3}^{\prime}\right) & =D_{k} \cap w_{4 n+3}^{\prime}=w_{4(n-1)+3}^{\prime} \\
\Delta\left(u_{4 n+1}^{\prime}\right) & =D_{k} \cap u_{4 n+1}^{\prime}=D_{k} \cap\left(B_{k}^{\prime} \cap w_{4 n+3}^{\prime}\right)=B_{k}^{\prime} \cap\left(D_{k} \cap w_{4 n+3}^{\prime}\right) \\
& =B_{k}^{\prime} \cap w_{4(n-1)+3}^{\prime}=u_{4(n-1)+1}^{\prime}, \Delta\left(v_{4 n+1}^{\prime}\right)=v_{4(n-1)+1}^{\prime}
\end{aligned}
$$

If

$$
\begin{aligned}
& F_{n}=U_{4 n}(p t) w_{3}^{\prime}+U_{4 n+2}(p t) u_{1}^{\prime}+U_{4 n+2}(p t) v_{1}^{\prime} \\
& F_{n}^{\prime}=U_{4 n-2}(p t) w_{3}^{\prime}+U_{4 n}(p t) u_{1}^{\prime}+U_{4 n}(p t) v_{1}^{\prime}
\end{aligned}
$$


then we have:

LEMMA 3.3. The following sequences are exact:

$$
\begin{aligned}
& 0 \rightarrow F_{n} \rightarrow U_{4 n+3}\left(B \Gamma_{k}\right) \stackrel{\Delta}{\rightarrow} U_{4(n-1)+3}\left(B \Gamma_{k}\right) \rightarrow 0, \\
& 0 \rightarrow F_{n}^{\prime} \rightarrow U_{4 n+1}\left(B \Gamma_{k}\right) \stackrel{\Delta}{\rightarrow} U_{4(n-1)+1}\left(B \Gamma_{k}\right) \rightarrow 0 .
\end{aligned}
$$

Proof. The proof is similar to that of Lemma 2.7 .

It remains to calculate the orders of the generators.

THEOREM 3.4. We have: ord $w_{4 n+3}^{\prime}=2^{2 n+k}, n \geq 0$.

Proof. We have $0=T_{k}\left(D_{k}\right)=2^{k} D_{k}+H\left(D_{k}\right) D_{k}^{2}$ and then $0=$ $\left(2^{k} D_{k}+H\left(D_{k}\right) D_{k}^{2}\right) \cap w_{7}=2^{k} w_{3}$ because: $D_{k}^{2} \cap w_{7} \in \widetilde{U}_{-1}\left(B \Gamma_{k}\right)=0$. Now if $\mu^{\prime}$ is the edge homomorphism: $U_{3}\left(B \Gamma_{k}\right) \rightarrow H_{3}\left(B \Gamma_{k}\right)=\mathbb{Z}_{2} k$ then we have $\mu^{\prime}\left(w_{3}^{\prime}\right)=1 \in \mathbb{Z}_{2} k$ and consequently $2^{k-1} w_{3} \neq 0$. Then ord $w_{3}=2^{k}$.

Suppose that ord $w_{4 i+3}^{\prime}=2^{2 i+k}, 0 \leq i \leq n-1$. Then

$$
\begin{aligned}
0= & T_{k}\left(D_{k}\right) \cap w_{4 n+7}^{\prime}=2^{k} w_{4 n+3}^{\prime}+2^{k-2} \lambda_{2}^{\prime} w_{4(n-1)+3}^{\prime} \\
& +\cdots+2^{k-i} \lambda_{i}^{\prime} w_{4(n-1)+3}^{\prime}+\cdots+2 \lambda_{k-1}^{\prime} w_{4(n-k+2)+3}^{\prime} \\
& +\lambda_{k}^{\prime} w_{4(n-k+1)+3}^{\prime}+\cdots+\lambda_{m}^{\prime} w_{4(n-m+1)+3}^{\prime}+\cdots,
\end{aligned}
$$

the number of non-zero elements in this sum being finite. If $3 \leq$ $i \leq k-1$ we have $2^{2 n-1+k-i} w_{4(n-i+1)+3}^{\prime}=0$ because ord $w_{4(n-i+1)+3}^{\prime}=$ $2^{2(n-i+1)+k}$ and $2(n-i+1)+k \leq 2 n-1+k-i$ since $i \geq 3$. If $m \geq k(\geq 3)$ we have $2^{2 n-1} w_{4(n-m+1)+3}^{\prime}=0$ because ord $w_{4(n-m+1)+3}^{\prime}=2^{2(n-m+1)+k}$ and $2(n-m+1)+k \leq 2 n-1$ since $k \leq m \leq 2 m-3$. It follows that $2^{2 n-1+k} w_{4 n+3}^{\prime}+2^{2 n-3+k} \lambda_{2}^{\prime} w_{4 n-1}^{\prime}=0$. Now $2^{2 n-3+k} w_{4 n-1}^{\prime} \neq 0$ because ord $w_{4 n-1}^{\prime}=2^{2 n-2+k}$; since $\lambda_{2}^{\prime} \notin 2 U^{-4}(p t)$ we have $2^{2 n-3+k} \lambda_{2}^{\prime} w_{4 n-1}^{\prime} \neq$ 0 (see 2.5). Hence

$$
2^{2 n-1+k} w_{4 n+3}^{\prime} \neq 0 \text { and } 2^{2 n+k} w_{4 n+3}^{\prime}=-2^{2(n-1)+k} \lambda_{2}^{\prime} w_{4 n-1}^{\prime}=0 .
$$

We have proved that ord $w_{4 n+3}=2^{2 n+k}$.

TheOREM 3.5. We have: ord $u_{4 n+1}^{\prime}=$ ord $v_{4 n+1}^{\prime}=2^{n+1}, n \geq 0$, which are therefore independent of $k$.

Proof. The proof of 3.5 is based on Theorem 3.2 and Lemma 3.3 and is exactly the same as the one of Theorem 2.8 . 


\section{REFERENCES}

[1] J. F. Adams, Stable Homotopy and Generalized Homology, University of Chicago Mathematics Lecture Notes, 1971.

[2] J. M. Boardman, Stable Homotopy Theory, Mimeographed notes, Chapter V, VI, Warwick, 1966.

[3] P. E. Conner and E. E. Floyd, Differentiable Periodic Maps, Springer, 1964.

[4] _ Periodic maps which preserve a complex structure, Bull. Amer. Math. Soc., 70 (1964), 574-579.

[5] _ Torsion in SU-bordism, Memoirs Amer. Math. Soc., 60 (1966).

[6] A. Mesnaoui, Unitary cobordism of classifying spaces of quaternion groups, to be published.

[7] D. Pitt, On the complex bordism of the generalized quaternion groups, J. London Math. Soc., 16 (1977), 142-148.

[8] R. M. Switzer, Algebraic Topology, Homotopy and Homology, Vol. 2.2, SpringerVerlag, 1975.

[9] R. H. Szczarba, On tangent bundles of fibre spaces and quotient spaces, Amer. J. Math., 86 (1964), 685-697.

[10] G. Wilson, K-theory invariants for unitary G-bordism, Quart. J. Math., Oxford, 24 (1973), 499-526.

Received October 5, 1986 and, in revised form, August 15, 1988.

UNIVERSITÉ MOHAMMEd V

B. P. 1014

RABAT, MOROCCO 



\section{PACIFIC JOURNAL OF MATHEMATICS EDITORS}

\author{
V. S. VARADARAJAN \\ (Managing Editor) \\ University of California \\ Los Angeles, CA 90024-1555-05 \\ Herbert Clemens \\ University of Utah \\ Salt Lake City, UT 84112 \\ ThOMAS ENRIGHT \\ University of California, San Diego \\ La Jolla, CA 92093
}

\section{R. FINN}

Stanford University

Stanford, CA 94305

HeRmann FlaschKa

University of Arizona

Tucson, AZ 85721

VAUGHAN F. R. JONES

University of California

Berkeley, CA 94720

Steven KerckhofF

Stanford University

Stanford, CA 94305

\author{
RobION KIRBY \\ University of California \\ Berkeley, CA 94720 \\ C. C. Moore \\ University of California \\ Berkeley, CA 94720 \\ HAROLD STARK \\ University of California, San Diego \\ La Jolla, CA 92093
}

\section{ASSOCIATE EDITORS}
R. ARENS
E. F. BECKenBach
B. H. NeumanN
F. WOLF
K. YoshidA (1906-1982)
(1904-1989)

\section{SUPPORTING INSTITUTIONS}

UNIVERSITY OF ARIZONA

UNIVERSITY OF BRITISH COLUMBIA

CALIFORNIA INSTITUTE OF TECHNOLOGY

UNIVERSITY OF CALIFORNIA

MONTANA STATE UNIVERSITY

UNIVERSITY OF NEVADA, RENO

NEW MEXICO STATE UNIVERSITY

OREGON STATE UNIVERSITY
UNIVERSITY OF OREGON

UNIVERSITY OF SOUTHERN CALIFORNIA

STANFORD UNIVERSITY

UNIVERSITY OF HAWAII

UNIVERSITY OF TOKYO

UNIVERSITY OF UTAH

WASHINGTON STATE UNIVERSITY

UNIVERSITY OF WASHINGTON 


\section{Pacific Journal of Mathematics}

Vol. 142, No. $1 \quad$ January, 1990

Marco Andreatta, Mauro Beltrametti and Andrew Sommese, Generic properties of the adjuction mapping for singular surfaces and applications

Chen-Lian Chuang and Pjek-Hwee Lee, On regular subdirect products of

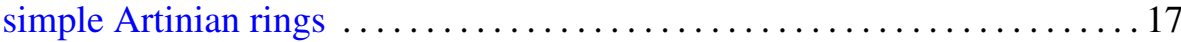

Fernando Giménez and Vicente Miquel Molina, Volume estimates for real hypersurfaces of a Kaehler manifold with strictly positive holomorphic sectional and antiholomorphic Ricci curvatures $\ldots \ldots \ldots \ldots \ldots \ldots 23$

Richard J. Griego and Andrzej Korzeniowski, Asymptotics for certain Wiener integrals associated with higher order differential operators

Abdeslam Mesnaoui, Unitary bordism of classifying spaces of quaternion

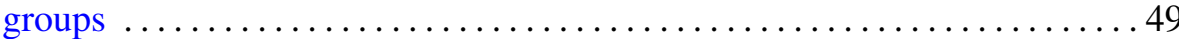

Abdeslam Mesnaoui, Unitary cobordism of classifying spaces of quaternion

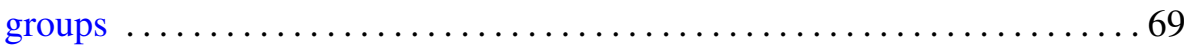

Jesper M. Møller, On equivariant function spaces $\ldots \ldots \ldots \ldots \ldots \ldots \ldots \ldots$

Bassam Nassrallah, A $q$-analogue of Appell's $F_{1}$ function, its integral representation and transformations

Peter A Ohring, Solvability of invariant differential operators on metabelian

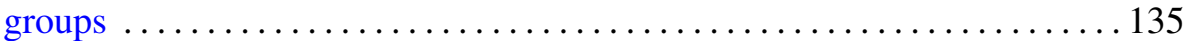

Athanase Papadopoulos and R. C. Penner, Enumerating pseudo-Anosov foliations

Ti-Jun Xiao and Liang Jin, On complete second order linear differential

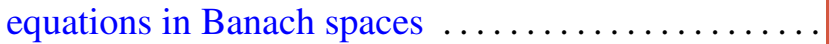

Carl Widland and Robert F. Lax, Weierstrass points on Gorenstein curves 